\title{
Broadband imaging and narrowband polarimetry of comet 73P/Schwassmann-Wachmann 3, components B and C, on $3,4,8$, and 9 May 2006 ${ }^{\star}$
}

\author{
T. Bonev ${ }^{1}$, H. Boehnhardt ${ }^{2}$, and G. Borisov ${ }^{1}$ \\ ${ }^{1}$ Institute of Astronomy, Bulgarian Academy of Sciences, Tsarigradsko chaussee 72, 1421 Sofia, Bulgaria \\ e-mail: tbonev@astro.bas.bg \\ 2 Max-Planck-Institut für Sonnensystemforschung, Max-Planck-Str. 2, 37191 Katlenburg-Lindau, Germany \\ e-mail: boehnhardt@mps .mpg.de
}

Received 22 August 2007 / Accepted 14 November 2007

\begin{abstract}
Context. The Jupiter family comet 73P/Schwassmann-Wachmann 3 (SW3) split into several pieces in 1995. Some of the original fragments were observed during the next apparition of the comet in 1999-2001. The last return of the comet in 2005-2006 was accompanied by tremendous further splitting of some SW3 components - in particular component B - into a large number of subfragments.

Aims. We present observations of components B and C during their closest approach to Earth in the first half of May 2006. These results aim at characterizing the properties of dust particles released from the fragments of comet SW3 and at identifying dusty and gaseous structures in the comae and tail regions of components $\mathrm{B}$ and $\mathrm{C}$, which could be useful for conclusions about the presence of active regions and break-up events of the components.

Methods. We used narrowband imaging polarimetry and an analysis of the broadband surface brightness for the characterization of the dust comae of the SW3 components. Coma and tail structures in components B and C were enhanced by numerical methods.

Results. The degree of linear polarization of components $\mathrm{B}$ and $\mathrm{C}$ follows the empirical phase-angle dependence as derived from other comets. Fragment B was found in outburst on May 8. The brightness outburst was accompanied by changes in the shape of the coma. During the outburst, the spatial distribution of the linear polarization showed some peculiarities that had disappeared again on May 9. Arc-like signatures of a temporary nature, typical of fragmentation events, are seen in broadband filter images of component B on 3, 8, and 9 May 2006. The secondary dust tail, seen in component B on 3 and 4 May 2006, marks the synchrone of an earlier splitting event around 25 April 2006. Fan-like coma structures are found in the sunward coma hemisphere of components B and C that might be related to localized enhanced activity on the rotating nuclei.
\end{abstract}

Key words. comets: general - comets: individual: 73P

\section{Introduction}

Comet 73P/Schwassmann-Wachmann 3 (hereafter 73P or SW3) is one of the most intriguing examples of fragmenting comets. First reports of unusual activity by the comet came from radio observations of $\mathrm{OH}$ in September 1995 (Crovisier et al. 1995). In December 1995, simultaneous $R$ band and thermal infrared images (Boehnhardt et al. 1995) revealed at least four fragments in the coma of the comet. Combined analysis of $\mathrm{OH}$ measurements and visual light curves led Crovisier et al. (1996) to the conclusion that the comet experienced sustained activity lasting for several months. During its apparition in 2001, the SW3 components appeared already well-separated in the sky and looked like individual comets with a normal and continuous evolution of brightness (at a higher level though) and no exceptional events (Boehnhardt et al. 2002; Boehnhardt 2004). Sekanina (2005) analyzed the correlation of outbursts and fragmentation events and used his concept of cascading fragmentation to make predictions for the positions of the fragments in 2006.

The recent apparition of $73 \mathrm{P}$ was very favorable for high spatial-resolution imaging. In May 2006 the SW3 components

* Based on observations performed at the National Astronomical Observatory, Rozhen, Bulgaria. approached Earth to distances $<0.1$ AU. Months before, the comet had started to show exceptional variability. Especially active were fragments $B$ and $G$, which experienced several events of further splitting into smaller pieces. Photometrical and positional measurements of the subfragments were reported by Birtwhistle \& Christensen (2006), Christensen et al. (2006), Christensen (2006), and Hasubick \& Nagai (2006). Weaver et al. (2006) used observations obtained with the Hubble Space Telescope to analyze the continuing disintegration of fragments $\mathrm{B}$ and $\mathrm{G}$, and detected a swarm of mini-comets around these fragments. No fragments could be detected around SW3 component C (Toth et al. 2006). Colom et al. (2006) measured $\mathrm{OH}$ production rates of fragments $\mathrm{B}$ and $\mathrm{C}$ over a time interval spanning about 2 months before the perihelion passage of the SW3 components. Villanueva et al. (2006) compared the volatile composition of fragments $\mathrm{B}$ and $\mathrm{C}$, and DiSanti et al. (2007) have revealed a depleted CO content in fragment C of SW3.

In this paper we present the analysis of $R$ filter images and narrow-band polarimetric images of SW3 components B and C obtained in the period 3-10 May 2006. In the next section we describe the instrumentation and provide details on the observations and the data reduction, emphasizing some non-standard 

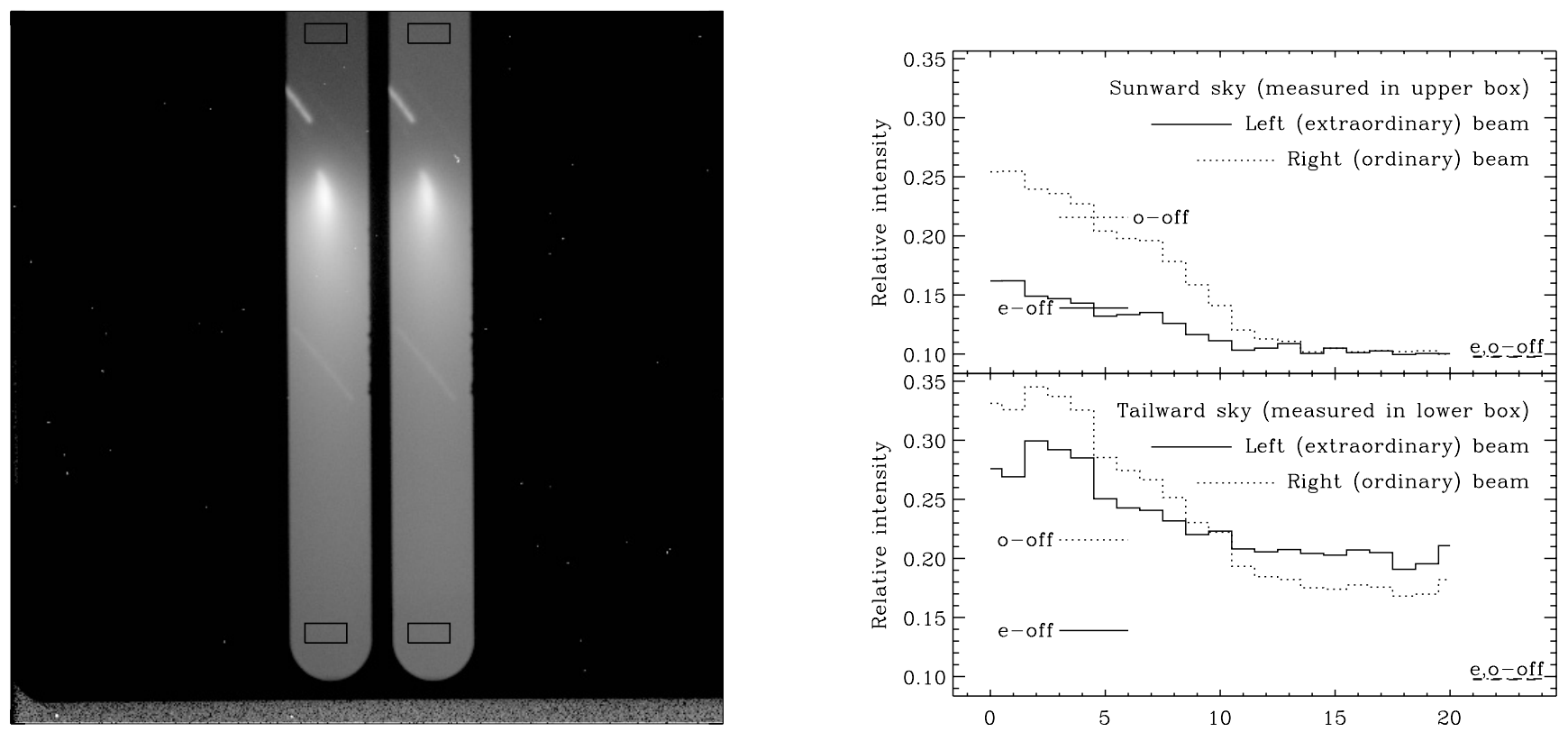

Fig. 1. Left panel: polarimetric image of SW3, component B on May 4. The left panel shows the image of the comet, decomposed into ordinary (right strip) and extraordinary (left strip) components by the Wollaston prism. The rectangular boxes at the top and bottom of the strips show the locations where sky measurements are taken. The instrument was rotated to bring the long side of the polarimetric strip mask perpendicular to the scattering plane of the dust in the coma. Orientation is as on sky with Sun direction up. Right panel, top: sky values measured sunward of the nucleus (boxes at the top of strip mask image) are presented versus sequential number of the image. Full line: perpendicular polarization, dotted line: parallel polarization in respect to the light scattering plane. Right panel, bottom: same as in the top right panel, but now measured tailward from the nucleus (boxes at the bottom of strip mask image). In both panels the horizontal lines centered between image 4 and 5 indicate the background level obtained from quasi-simulatenous exposures taken about 1 degree sunward of the comet. These levels are labeled with "o-off" and "e-off" for the measurement of the ordinary and extraordinary beam, respectively. The background measurements taken far from the comet at the end of the observing sequence are labeled with "e,o-off". In this latter case the background levels in the ordinary and extraordinary beams are practically identical (dark sky, see text).

steps in the reduction of the polarimetric images. Further, we present the results in the form of contour maps and profiles of the $R$ band images, we analyze the structures in the coma of fragments $\mathrm{B}$ and $\mathrm{C}$, and show profiles of the spatial distribution of the degree of linear polarization. We explain the observed trend of the decreasing polarization degree with increasing distance from the fragments by the influence of molecular emissions. Deviations in this trend are related to different properties of the dust released during an outburst observed in fragment B. Finally, we give a brief summary of our results.

\section{Observations and data reduction}

\subsection{Instrumentation and observing schedule}

The observations were made with FoReRo2, the 2-channel Focal Reducer Rozhen, mounted at the 2-m Ritchey-Chrétien-Coude telescope of the National Astronomical Observatory in Rozhen, Bulgaria. Details for this instrument are described by Jockers (1997b). FoReRo2 reduces the focal length of the telescope from $16000 \mathrm{~mm}$ to $5600 \mathrm{~mm}$. This paper is based on data obtained in the red arm of the focal reducer. The detector in the red channel is an $\mathrm{LN}_{2}$ cooled CCD camera of type VersArray $512 \mathrm{~B}$, which provides a field of view $7.5 \times 7 ! 5$. The scale is $0.89 /$ pixel, adapted to the mean seeing at Rozhen. Polarimetric imaging with FoReRo2 was performed by placing a Wollaston prism in the parallel beam of the focal reducer. The position of the Wollaston is close to the exit pupil of the field lens collimator. The Wollaston prism separates the parallel beam of incoming light in two linearly polarized components, which are mutually orthogonal and which are usually referred to as the ordinary and extraordinary beams. The deviation angle between the two emerging beams is 43.6 , corresponding to a separation of 74 pixels on the CCD chip and equivalent to $66^{\prime \prime}$ on the sky. To avoid overlap of the two polarized beams, a strip mask of width $3.6 \mathrm{~mm}\left(=46^{\prime \prime}\right)$ is inserted in the intermediate focal plane. The length of the mask extends over the whole field of view. The position angle of the telescope was rotated during the observations to align the images produced by the Wollaston prism with the projected direction of the scattering plane. An example of an image obtained in polarization mode is shown in the left panel of Fig. 1.

For the SW3 observations, we used the FoReRo2 in filter imaging and imaging polarimetry mode. The observing schedule is summarized in Table 1, together with some basic geometric information.

\subsection{Broadband filter imaging}

Initially, broadband images were only intended for detecting and centering the comet in the field of view. We planned to continue with cometary narrowband continuum filters, sensing the solar light scattered by cometary dust particles, but problems with tracking the fast-moving comet during long exposures smeared its image on the CCD. For that reason and because of nonphotometric weather conditions, we decided to use Bessel $R$ filter exposures for a more extensive normal imaging monitoring of the SW3 components. Typical exposure times with this filter were between 10 and $30 \mathrm{~s}$, short enough to keep the tracking errors within reasonable limits. After bias-subtraction and flatfielding, the images were calibrated by differential photometry, 
Table 1. Geometrical conditions during the observations.

\begin{tabular}{|c|c|c|c|c|c|c|c|c|c|}
\hline $\begin{array}{c}\text { Day of May } 2006 \\
\text { from - to } \\
(\mathrm{UT})-(\mathrm{UT}) \\
\end{array}$ & $\begin{array}{l}\text { Observ. } \\
\text { mode }^{c}\end{array}$ & $\begin{array}{l}r^{a} \\
(\mathrm{AU}) \\
\end{array}$ & $(\mathrm{AU})$ & $\begin{array}{l}\text { Phase } \\
\text { angle } \\
\left({ }^{\circ}\right) \\
\end{array}$ & $\begin{array}{c}\text { Mean } \\
\mathrm{PA}^{d} \\
\left({ }^{\circ}\right) \\
\end{array}$ & $\begin{array}{c}\text { Rotator } \\
\text { angle } \\
\left({ }^{\circ}\right) \\
\end{array}$ & $\begin{array}{c}\begin{array}{c}\text { Scale at } \\
\text { comet } \\
\left(\mathrm{km} \mathrm{px}^{-1}\right)\end{array} \\
\end{array}$ & $\begin{array}{r}\text { Total } \\
\text { exp.time } \\
\text { (seconds) } \\
\end{array}$ & $\begin{array}{c}\text { Mean } \\
\text { signal }^{f} \\
10^{6} \mathrm{ADU} \\
\end{array}$ \\
\hline \multicolumn{10}{|c|}{ Fragment B } \\
\hline $3.972-3.985$ & I & 1.068 & $0.10 \overline{9}$ & 54.6 & 212.0 & 0.0 & 79.3 & 520 & 27.0 \\
\hline $4.854-4.863$ & I & 1.062 & 0.104 & 56.6 & 213.9 & -37.4 & 75.6 & 600 & 18.1 \\
\hline $4.893-5.053$ & $\mathrm{P}$ & 1.062 & 0.103 & 56.6 & 213.9 & -37.4 & 75.6 & 11040 & $9.8 / 7.7$ \\
\hline $8.883-8.896$ & I & 1.036 & 0.081 & 68.4 & 227.4 & -47.4 & 58.9 & 282 & 148.8 \\
\hline $8.965-8.994$ & $\mathrm{P}$ & 1.036 & 0.080 & 68.4 & 227.4 & -47.4 & 58.9 & 1500 & $31.6 / 22.1$ \\
\hline $9.853-9.866$ & I & 1.031 & 0.076 & 72.0 & 231.8 & -56.5 & 55.3 & 270 & 31.2 \\
\hline $9.870-9.914$ & $\mathrm{P}$ & 1.031 & 0.076 & 72.0 & 231.8 & -56.5 & 55.3 & 2910 & $30.2 / 20.5$ \\
\hline \multicolumn{10}{|c|}{ Fragment C } \\
\hline $4.070-4.076$ & I & 1.061 & $0.10 \overline{7}$ & 57.9 & 225.2 & 0.0 & 77.8 & 280 & 22.0 \\
\hline $5.095-5.105$ & I & 1.054 & 0.101 & 60.5 & 227.7 & -37.4 & 73.4 & 420 & 55.3 \\
\hline $9.047-9.056$ & I & 1.030 & 0.084 & 73.8 & 240.7 & -47.4 & 61.1 & 190 & 25.5 \\
\hline $9.934-9.939$ & I & 1.024 & 0.081 & 77.2 & 243.7 & -56.5 & 58.9 & 240 & 23.4 \\
\hline $9.944-10.053$ & $\mathrm{P}$ & 1.024 & 0.081 & 77.4 & 243.7 & -56.5 & 58.9 & 6100 & $21.6 / 14.8$ \\
\hline
\end{tabular}

${ }^{a}$ Heliocentric distance; ${ }^{b}$ geocentric distance; ${ }^{c} \mathrm{I}=$ imaging, $\mathrm{P}=$ polarimetric imaging; ${ }^{d}$ extended Sun-fragment radius vector, defines also the scattering plane; ${ }^{e}$ The values give the angle between North and the $+Y$ axis. Sign minus $(-)$ means that North is rotated clockwise; ${ }^{f}$ measured in circular aperture of radius $10^{\prime \prime}$.

which makes the result independent from the changing photometric conditions. Field stars in the comet exposures are used as brightness standards for flux calibration (with $R$-magnitudes adopted from the USNO-A2.0 catalog). Despite the relatively low photometric accuracy of this catalog, its use may be justified here by the large number of stars per field, which in turn improves the scatter in the photometric results.

\subsection{Imaging polarimetry}

The polarimetric images were obtained through a narrowband red continuum filter (central wavelength $=684 \mathrm{~nm}$, full width at half maximum transmission $=9 \mathrm{~nm}$ ). In order to increase the signal to noise ratio (SNR), sequences of images of the SW3 components were obtained. Data for these sequences are given in Table 1.

The images were bias-subtracted and flat-fielded with master flats obtained without the strip mask. In the next step of the reduction process, the images from the ordinary and extraordinary beams were treated separately. Subimages of width $700 \mathrm{~km}$ $(+/-350 \mathrm{~km}$ to the left and to the right from the photocenter of the components) were extracted for each beam, aligned with subpixel accuracy and combined to a single averaged image.

One of the advantages of polarimetric imaging with a Wollaston prism is the simulatenous measurements of two mutually orthogonal components of polarized light. This makes the derivation of the degree of polarization insensitive to changes in the atmospheric transparency during the observations. However, the observations of comet $73 \mathrm{P}$ were obtained in bright time, i.e. when scattered moonlight polarized the sky background. The sky illumination conditions, both background level and polarization, were strongly variable, depending on the height of the Moon above horizon. The changing sky background polarization is illustrated in Fig. 1. The boxes at the top and bottom of the strip masks show the windows in which the sky was measured for our analysis. During the acquisition of a sequence of images, the telescope was moved from the comet to a sky position more than $1^{\circ}$ away from the SW3 components in order to measure the sky background. The right panel in Fig. 1 shows the results of these measurements, together with the background levels as determined from the "sky window boxes" in the corresponding strip mask image. These data allow two important conclusions: first, they show that the gradients of the decreasing sky background are different for both polarization beams; i.e., the sky polarization varies with changing sky brightness. Second, a comparison of the background level in the sunward and tailward "sky window boxes" with the measurements on clear sky shows that the latter agree with the background level in the sunward (upper) "sky window boxes". Because of this and the temporal variability of the sky level, we used the sunward "sky window boxes" of each mask image for estimating of the background sky in the SW3 polarimetry exposures.

Apart from the temporal variations in the background sky, the polarization measurements are also affected by spatial variations due to the instrumental polarization. For an estimation of instrument polarization we observed unpolarized standard stars at different positions along the polarimetric strip mask, i.e. HD 185395 from the list of Serkowski (1974), and HD 214923 and HD 195068 taken from Appenzeller (1966). The results of these measurements are shown in Fig. 2.

The data of 4 May 2006 (symbol = triangle) clearly show that the instrumental polarization decreases linearly with increasing vertical distance in the strip mask. On 8 and 9 May (symbols = boxes and crosses, respectively), we measured the standards only in a position close to the center. It can be assumed that the instrument polarization does not change with time. The different zero points of the instrument polarization, seen for the unpolarized stars in Fig. 2, is due to the different position angles of the polarization strip mask in the sky. To correct for the instrument polarization, we thus obtained its spatial gradient from the 4 May data and applied it to all polarization images of the SW3 components.

For observations of solar system objects, the light-scattering plane can be assumed to coincide with the plane Sun-objectEarth. The polarization degree $P$ is defined with reference to this scattering plane as

$$
P=\frac{I_{\perp}-I_{\|}}{I_{\perp}+I_{\|}}
$$

where $I_{\perp}$ and $I_{\|}$are the (relative) fluxes of the object in the beam that is polarized perpendicular and parallel to the 


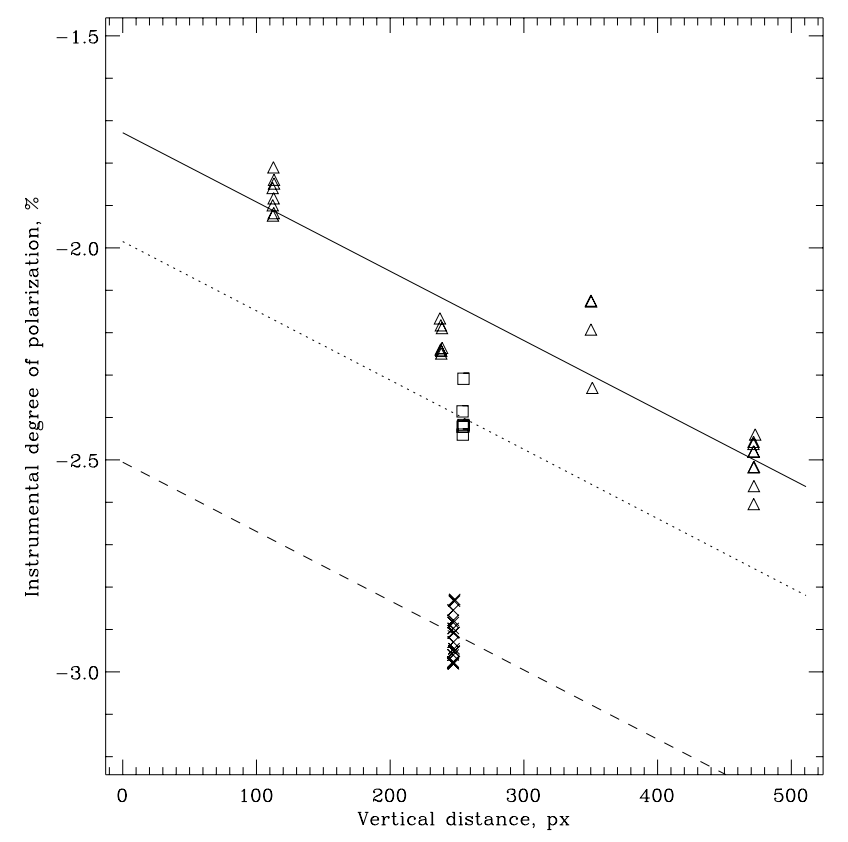

Fig. 2. Field dependence of the instrument polarization.

scattering plane, respectively. We used the known orientation of the scattering plane and rotated the instrument accordingly. Thus we aligned the Wollaston beams (e.g. the ordinary one) along the plane of light-scattering "Sun-comet-Earth". The position angle of the scattering plane projected in the sky changed from one night to the next. Moreover, due to the large angular distance of the two SW3 components in the sky, it was different for both fragments in the same night. In order to apply the same instrument polarization correction per night for both fragments, we rotated the instrument to an angle intermediate between the nominal scattering angles for components B and C.

However, due to this intermediate orientation of the polarizing optics, the parallel and perpendicular components of the scattered light no longer coincide with the ordinary and extraordinary beams of the Wollaston. Hence, the polarization degree calculated via Eq. (1) needs to be corrected. The relation between the two components of the measured irradiance, $I_{\mathrm{o}}$ and $I_{\mathrm{e}}$, (flux of ordinary (o) and extraordinary (e) beams), and the perpendicular and parallel components of scattered light from the comet, $I_{\perp}$ and $I_{\|}$, is given by Malus' law:

$I_{\mathrm{e}}=\left(I_{\perp}-I_{\|}\right) \times \cos ^{2} \theta+I_{\|}$and $I_{\mathrm{o}}=\left(I_{\|}-I_{\perp}\right) \times \cos ^{2} \theta+I_{\perp}$,

where $\theta$ is the angle between the plane of principal transmittance of the corresponding beam of the Wollaston prism and the plane of the electric field vector of the incident beam. Using relations (1) and (2), we obtain the final formula for calculating the degree of polarization $\mathrm{P}$ :

$$
P=\frac{I_{\mathrm{e}}-I_{\mathrm{o}}}{\left(I_{\mathrm{e}}+I_{\mathrm{o}}\right) \times\left(2 \times \cos ^{2} \theta-1\right)} .
$$

Note that, during the observations, the rotator was turned manually to the desired angle, but the values listed in Table 1 were derived from the astrometric solutions of the images.

\section{Results}

\subsection{Overall appearance of the comae from broadband imaging}

Isophote patterns: For the preparation of surface brightness maps of both SW3 components, the $R$ filter images of each particular night were centered on the central brightness peak in the coma (the likely nucleus position) and median-averaged. This procedure increases the signal-to-noise level of the coma isophotes and widely surpresses the signal of background object shining through the comae. Figure 3 shows the surface brightness distribution of fragment B. Obviously, the comet experienced considerable changes in its coma isophotes between May 4 (second panel) and May 8 (third panel). The morphology of the brightness distribution changed from an elongated shape to a more oval, inflated form. The concentration of the coma towards the nucleus has increased. The orientation of the tailward part turned closer to anti-solar direction, and an extended narrow feature appeared along its axis. Inflation of the coma is often seen in comets during phases of increased activity or brightness outbursts (Bonev et al. 2002). It is related to the production of increased amount of small dust particles moving radially outward with high velocities. The changed orientation of the tail is an additional argument in support of this idea. The narrow deformation of the coma in an antisolar direction could be caused by small particles with low outflow velocity. These particles can appear when large grains, having low velocities, split into smaller dust particles outside the gas-dust interaction region (Combi 1994). The increased concentration seen towards the nucleus suggests that, on 8 May 2006, component B was in an enhanced activity state. Further indications of this outburstlike event are described in Sect. 3.2.

In contrast to $\mathrm{B}$, the isophotes of component $\mathrm{C}$ did not change significantly during the observing period. The surface brightness maps of $\mathrm{C}$ are shown in Fig. 4.

Near-nucleus coma photometry: A quantitative measure of the variations in activity is given by the brightness of a comet, measured in a fixed area around its nucleus. Integrated magnitudes of fragments B and C are shown for two circular apertures in Table 2. The magnitude of $\mathrm{C}$ is relatively stable and does not change by more than \pm 0.3 mag. In contrast, component $B$ experienced a brightness outburst of about 3 mag. This outburst event was noted by several observers who monitored SW3 at that time (Lara et al. 2006).

Radial coma and Af $\rho$ profiles: Radial coma profiles were calculated by averaging the mean coma image in concentric rings of increasing radius centered on the central brightness peak in the coma. We also computed the aperture-integrated Af $\rho$ profiles of the comae of components B and C. The quantity Af $\rho$ was introduced by A'Hearn et al. (1984) as a measure of dust production in comets.

Figure 5 shows the mean brightness profiles of the comae of components $\mathrm{B}$ and $\mathrm{C}$, compared to the inversed radial distance law (dotted lines) expected for homogenous and isotropic outflow conditions. The profile of B follows closely the $1 / r$ lines before the outburst. On May 8 it is steeper at distances greater than thousand $\mathrm{km}$, and on the next day it returns to the $1 / r$ distribution. The mean radial profiles of fragment $\mathrm{C}$ (right panel in Fig. 5) did not change significantly during the observing period and closely resembled the inverse radial distance law. 

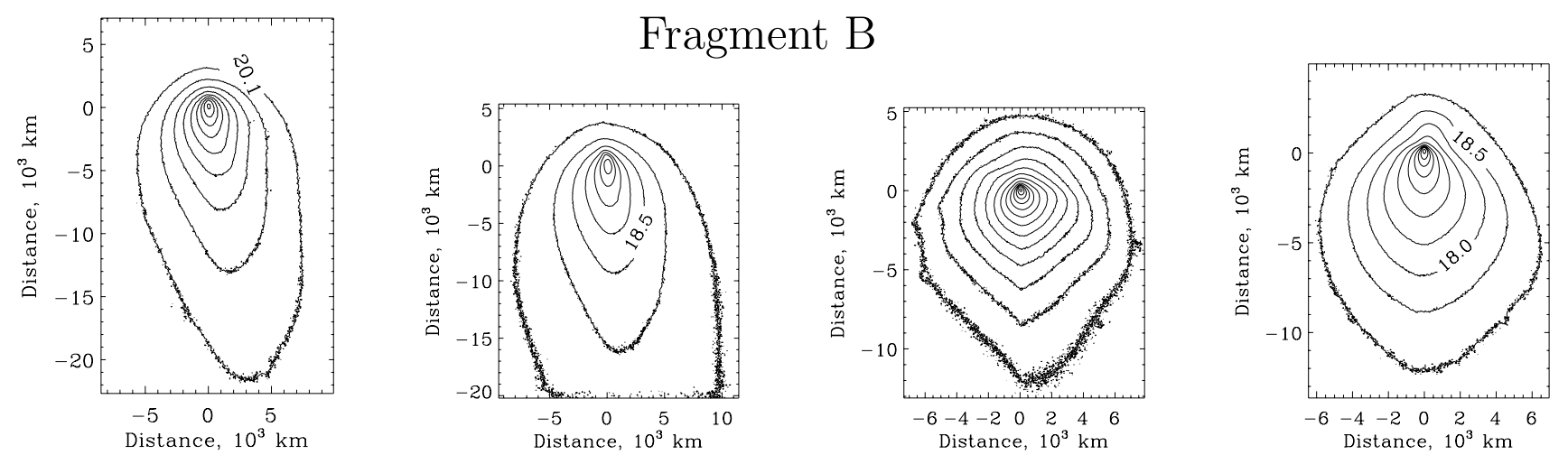

Fig. 3. Surface brightness distribution in SW3 component B. Maps of 3, 4, 8, and 9 May 2006, respectively, are shown from left to right. Orientation as in the sky with the Sun up. The outermost contours are taken at $1-\sigma$ above the sky background level.
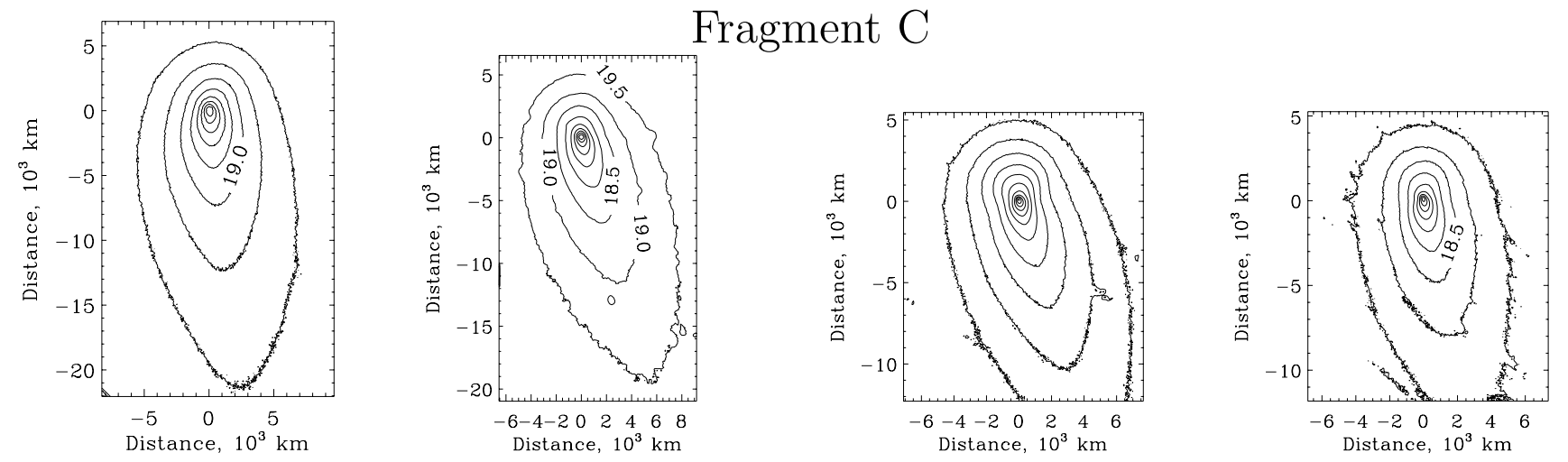

Fig. 4. Same as in Fig. 3 but for SW3 component C. The brightness distribution of the coma around this fragment does not show any significant changes.

Table 2. Total magnitude measurements of components B and C.

\begin{tabular}{cccccc}
\hline \hline \multicolumn{3}{c}{ Fragment B } & \multicolumn{3}{c}{ Fragment C } \\
Day of & \multicolumn{2}{c}{ Radius of aperture } & \multicolumn{2}{c}{ Day of } & \multicolumn{2}{c}{ Radius of aperture } \\
May 2006 & $10^{\prime \prime}$ & $20^{\prime \prime}$ & May 2006 & $10^{\prime \prime}$ & $20^{\prime \prime}$ \\
\hline 3.979 & 10.6 & 9.8 & 4.042 & 10.7 & 10.0 \\
4.854 & 10.6 & 9.6 & 5.042 & 10.1 & 9.4 \\
8.896 & 7.9 & 7.2 & 9.054 & 10.3 & 9.5 \\
9.858 & 9.2 & 8.5 & 9.936 & 10.4 & 9.5 \\
\hline
\end{tabular}

The influence of the outburst on the mean spatial distribution is furthermore illustrated by the $A f \rho$-profiles shown in Fig. 6. From 4 to 8 May 2006 the $A f \rho$-level increased by a factor of 10 . Moreover, the $A f \rho$-profile was almost independent of radial distance on 4 May which indicates that fragment B was in a quiescent phase at that time. Four days later, on 8 May, the profile shows a steeper decrease beyond about 1000 distance from the nucleus that had disappeared again on 9 May. The higher Af $\rho$-level in component B could be caused by an increased dust production rate, but it should not necessarily be proportional to the amplitude of the observed brightness outburst. Following the analysis of the fragmentation sequence of comet C/2001 A2 (LINEAR) (Sekanina et al. 2002), we could consider the outburst as indicating the release of subfragments that were rapidly disintegrating afterwards into fine dust $(\$ 1 \mu \mathrm{m})$ that scattered more efficiently in the spectral range of the filter used.

Sun-tail profiles: For the analysis of the dust distribution in the direction of the Sun and into the tail and to provide information

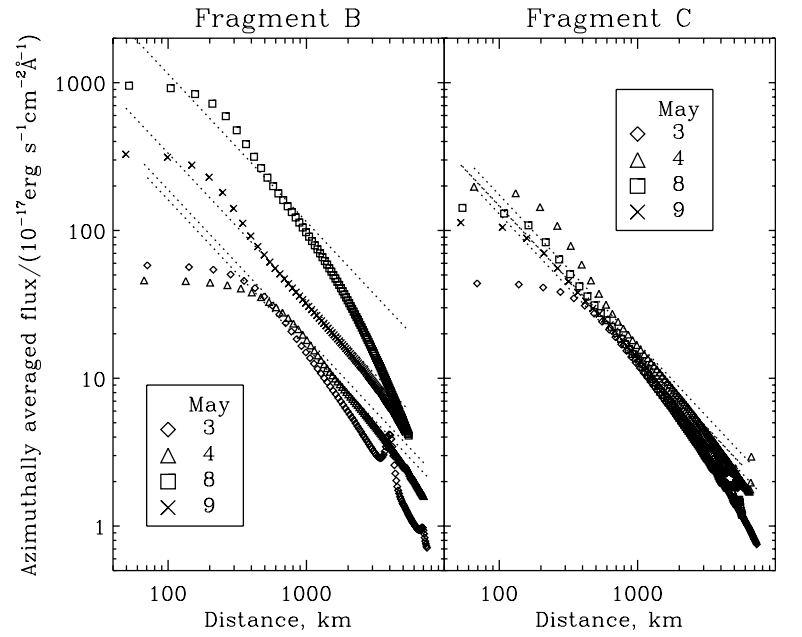

Fig. 5. Mean radial profiles of SW3 components B and C, derived from $R$ filter images. Four different symbols are used to distinguish between the profiles from the different observing epochs. Dotted lines indicate the $1 / \rho$ distribution, expected in the case of "stationary" radial outflow.

on the coma flux distribution that can be compared to the polarimetric measurements of the dust in the strip mask images, we extracted rectangular sections along the Sun-tail direction from the averaged filter images of the comae and of extension of the polarimetric strip mask pattern (width of $1000 \mathrm{~km}$ ). Integration over the strip width, i.e. perpendicular to the sun-comet line, resulted in the profiles shown in Fig. 7. 


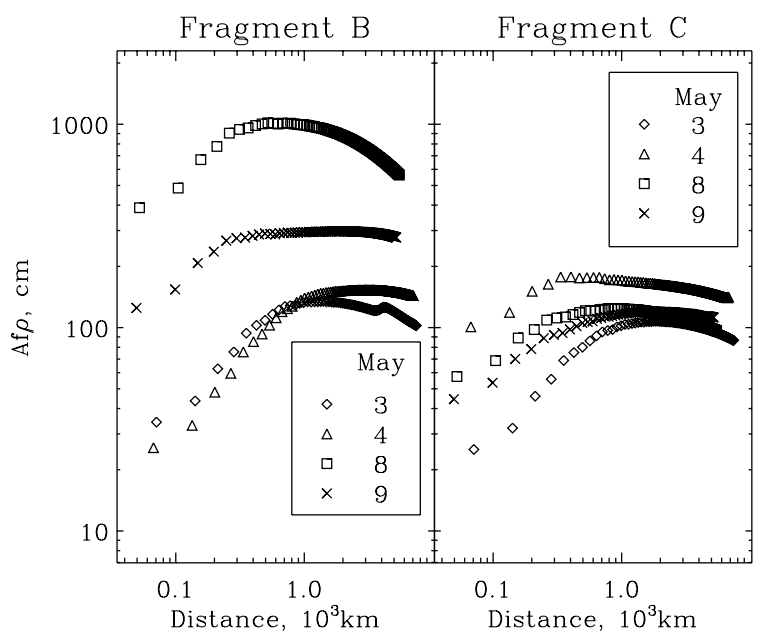

Fig. 6. Af $\rho$-profiles of SW3 components $\mathrm{B}$ and $\mathrm{C}$, derived from the $R$ filter images. Note the substantial increase in the $A f \rho$-level in component B on 8 May 2006, after an outburst of this fragment shortly before the measurement.

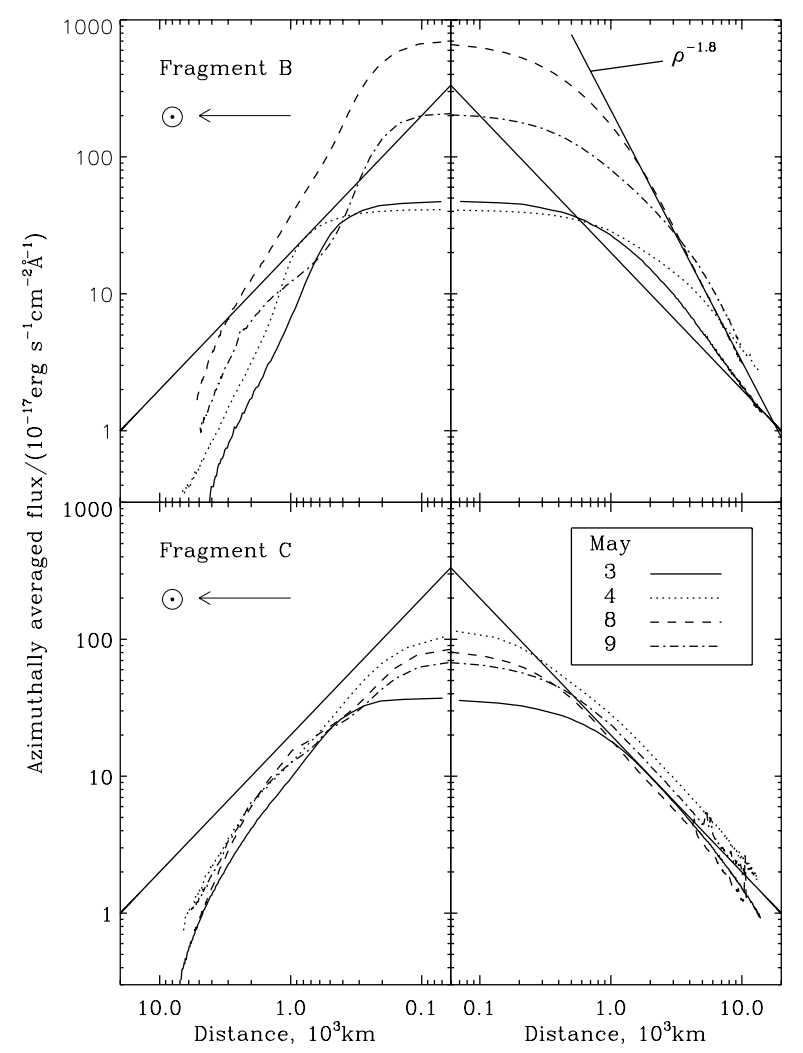

Fig. 7. Sun-tail profiles of the comae of SW 3 components B and C. In the left panels the profiles are plotted in the direction toward the Sun (top direction in Figs. 3 and 4). The right panels show the profiles in a tailward direction. As shown in the lower right subpanel, different line styles are used for the results from different observing nights. The symmetric continuous lines indicate a $1 / \rho$ profile in Sun and tailward directions. A profile $\propto \rho^{-1.8}$ fits best the tailward distribution of component B on 8 May 2006.

On 3 and 4 May 2006 the tailward profiles of component $\mathrm{B}$ resembled the $1 / \rho$ distribution, typical of the "stationary" (isotropic and homogenous) radial outflow of dust from a point source (upper panels in Fig. 7). The increased brightness on 8 May is accompanied by a steeper profile in the tail direction; i.e., from $1000-10000 \mathrm{~km}$ distance the profile slope is closely described by a power law with gradient -1.8. On 9 May 2006 the profile gradient became flatter again and it looks like component B was returning to a quiescent state. On 9 May 2006 the gradual decrease of the profile of B in sunward direction is disturbed by a step-like feature starting at around $1000 \mathrm{~km}$. The position of this feature corresponds to the elongated structure of the coma in this direction, clearly displayed in the fourth panel in Fig. 3, and it may be related to the sunward coma fan identified by the structure analysis of the images described in Sect. 3.2. As expected, the Sun-tail brightness profiles of component $\mathrm{C}$ (lower panels in Fig. 7) follow on the average a $1 / \rho$ distribution. However, on the sunward side, deviations that are similar to what are seen in component B on 9 May 2006 are apparent, which are related to localized sunward activity (fan structure; see Sect. 3.2).

\subsection{Coma structures}

Analysis method: For the analysis of structures inherent in the comae of the SW3 components B and C, we applied two standard enhancement techniques, i.e. adaptive Laplace filtering (Boehnhardt \& Birkle 1994) and radial renormalization (A'Hearn et al. 1986). The result frames and any structure therein were cross-checked by visual inspection of the flux-calibrated images for asymmetries and irregular coma isophotes, which can be considered to indicate the actual presence of the respective structures. Any structure found was characterized by its geometric extension (projected to the comet distance), near-nucleus position angle PA, and its curvature (see Tables 3 and 4).

Coma structures in component $\mathrm{C}$ (Table 3 and Fig. 8): Since the $R$ filter used for the coma imaging is most sensitive to dustreflected sunlight and coma gas contaminations are low (several weak bands of $\mathrm{NH}_{2}$ only), we attribute the coma structures identified and described below to dust phenomena in the comet. As outlined in Sect. 3.1, the overall coma isophotes of component C have a very regular shape and vary only slightly, in particular in the sunward hemisphere. Here, the structure's enhancement techniques reveal two short $(2500-5000 \mathrm{~km})$ and straight jets of variable intensity (FA and FB in Fig. 8). On 3, 8, and 9 May, structures FA and FB appear to be embedded in a common fanlike envelope (opening angle about $80-100^{\circ}$ ) pointing into the sunward coma hemisphere. Structure FA was brighter than structure FB. Variable intensities of the phenomenon from night to night are obvious. The FA-FB fan was weakest on 4 May, and FA was strongest on 8 and 9 May 2006. Given the short duration of our broadband imaging per night, nothing can be said about the short-term variability of the various coma structures.

The two structures FA and FB seen in the sunward coma hemisphere of component $\mathrm{C}$ seem to be related to temporarily enhanced local dust emission from one or two active regions on the rotating nucleus. Although the enhanced images suggest that FA and FB might be connected and in fact define the border limits of a projected dust emission cone in the coma produced by a single active region, it cannot be excluded that both structures are unrelated and represent dust emission from two different active regions on the nucleus. If the former interpretation is correct, it is likely that the rotation axis of the nucleus projects into the PA range of the FA-FB fan and the opening angle constrains the latitude of the underlying active region (medium latitude around $50^{\circ}$ for a spherical nucleus).

In the tailward side, only the long (>14000-35000 km) central part of the dust tail is found pointing approximately in the 
Table 3. Geometry of the main coma structures of dusty origin in the SW3 components B and $\mathrm{C}^{a}$.

\begin{tabular}{|c|c|c|c|c|c|}
\hline $\begin{array}{l}\text { SW3 } \\
\text { Comp }\end{array}$ & $\overline{\mathrm{IID}}$ & $\begin{array}{c}\text { PA (deg) } \\
\text { 3/4/8/9 May }\end{array}$ & $\begin{array}{c}\text { Curv } \\
\text { 3/4/8/9 May }\end{array}$ & $\begin{array}{l}\text { MinExt }(\mathrm{km}) \\
\text { 3/4/8/9 May }\end{array}$ & Comment \\
\hline $\bar{B}$ & FA & $\mathrm{nd} / \mathrm{nd} / 19 / 15$ & $\mathrm{nd} / \mathrm{nd} / \mathrm{S} / \mathrm{S}$ & $\mathrm{nd} / \mathrm{nd} / 4000 / 3300$ & continuous fan area? \\
\hline B & FB & $\mathrm{nd} / \mathrm{nd} / 54 / 62$ & $\mathrm{nd} / \mathrm{nd} / \mathrm{S} / \mathrm{S}$ & $\mathrm{nd} / \mathrm{nd} / 3500 / 3000$ & conti \\
\hline B & SP & $35 / 42 / \mathrm{nd} / \mathrm{nd}$ & $\mathrm{S} ? / \mathrm{CC} ? / \mathrm{nd} / \mathrm{nd}$ & $300 / 600 / \mathrm{nd} / \mathrm{nd}$ & sunward dust spike \\
\hline B & T1 & $216 / 221 / 226 / 236$ & $\mathrm{~S} / \mathrm{S} / \mathrm{S} / \mathrm{S}$ & $>28000 />22000 />16000 />14000$ & main dust tail \\
\hline B & $\mathrm{T} 2$ & 225/233/242?/nd & $\mathrm{CC} / \mathrm{CC} / \mathrm{S} ? / \mathrm{nd}$ & $21000 />23000 />16000 / \mathrm{nd}$ & sec. dust tail plus fragments \\
\hline $\mathrm{C}$ & FA & $9 / 27 / 28 / 42$ & $\mathrm{~S} / \mathrm{S} ? / \mathrm{S} / \mathrm{S}$ & $5000 / 3000 / 4500 / 3000$ & continuous fan area? \\
\hline $\mathrm{C}$ & FB & $52 / 78 / 85 / 105$ & $\mathrm{~S} / \mathrm{S} ? / \mathrm{S} / \mathrm{S}$ & $5000 / 3000 / 4000 / 2500$ & ontinuous fan area? \\
\hline $\mathrm{C}$ & $\mathrm{T}$ & $230 / 230 / 240 / 240$ & $\mathrm{~S} / \mathrm{S} / \mathrm{S} / \mathrm{S}$ & $>35000 />25000 />20500 />14000$ & main dust tail \\
\hline
\end{tabular}

${ }^{a}$ Notes: The table lists the comet component (SW3 Comp), the structure designation (ID), the near-nucleus position angle (PA; measured North over East), the curvature (Curv), and minimum extension (MinExt), plus some comments. In columns "PA", "Curv", "MinExt" the measurements for the four observing nights are listed, separated by " ". Abbreviations: $n d=$ not detected, $C C=$ counterclockwise, $S=$ straight. The various coma structures are identified in Figs. 8 and 9.

Table 4. Geometry of the arc structures in SW3 component B ${ }^{a}$.

\begin{tabular}{cccccl}
\hline \hline $\begin{array}{c}\text { Date } \\
(\mathrm{UT})\end{array}$ & $\begin{array}{c}\text { Orient } \\
(\mathrm{deg})\end{array}$ & Curv & $\begin{array}{c}\text { VerDist } \\
(\mathrm{km})\end{array}$ & $\begin{array}{c}\text { MinExt } \\
(\mathrm{km})\end{array}$ & Comment \\
\hline $03 / 05 / 2006$ & $125 / 325$ & TW & 1000 & $4000 / 5000$ & \\
$08 / 05 / 2006$ & $122 / \mathrm{nd}$ & $\mathrm{CC} / \mathrm{nd}$ & 1700 & $>12500 / \mathrm{nd}$ & single sided arc \\
& $142 / 322$ & $\mathrm{CC} / \mathrm{C}$ & 400 & $>17500 />13500$ & brighter western arc \\
$09 / 05 / 2006$ & $177 / 272$ & $\mathrm{CC} / \mathrm{C}$ & 1300 & $3000 / 2700$ & \\
& $142 / 322$ & $\mathrm{CC} / \mathrm{C}$ & $1000-3000$ & $>13000 />18000$ & $\begin{array}{l}\text { diffuse area inbetween } \\
\text { arc to 8500 km distance }\end{array}$ \\
\hline
\end{tabular}

${ }^{a}$ Notes: The table lists the observing date (Date), the orientation angle (Orient) measured North over East, the curvature (Curv), the distance of the arclet vertex from the nucleus (VerDist) and the minimum extension (MinExt) plus some comments. Abbreviations: $n d=$ not detected, $\mathrm{S}=$ straight, $\mathrm{C}=$ clockwise, $\mathrm{CC}=$ counterclockwise, $\mathrm{TW}=$ tailward. The various structures are identified in Fig. 9.
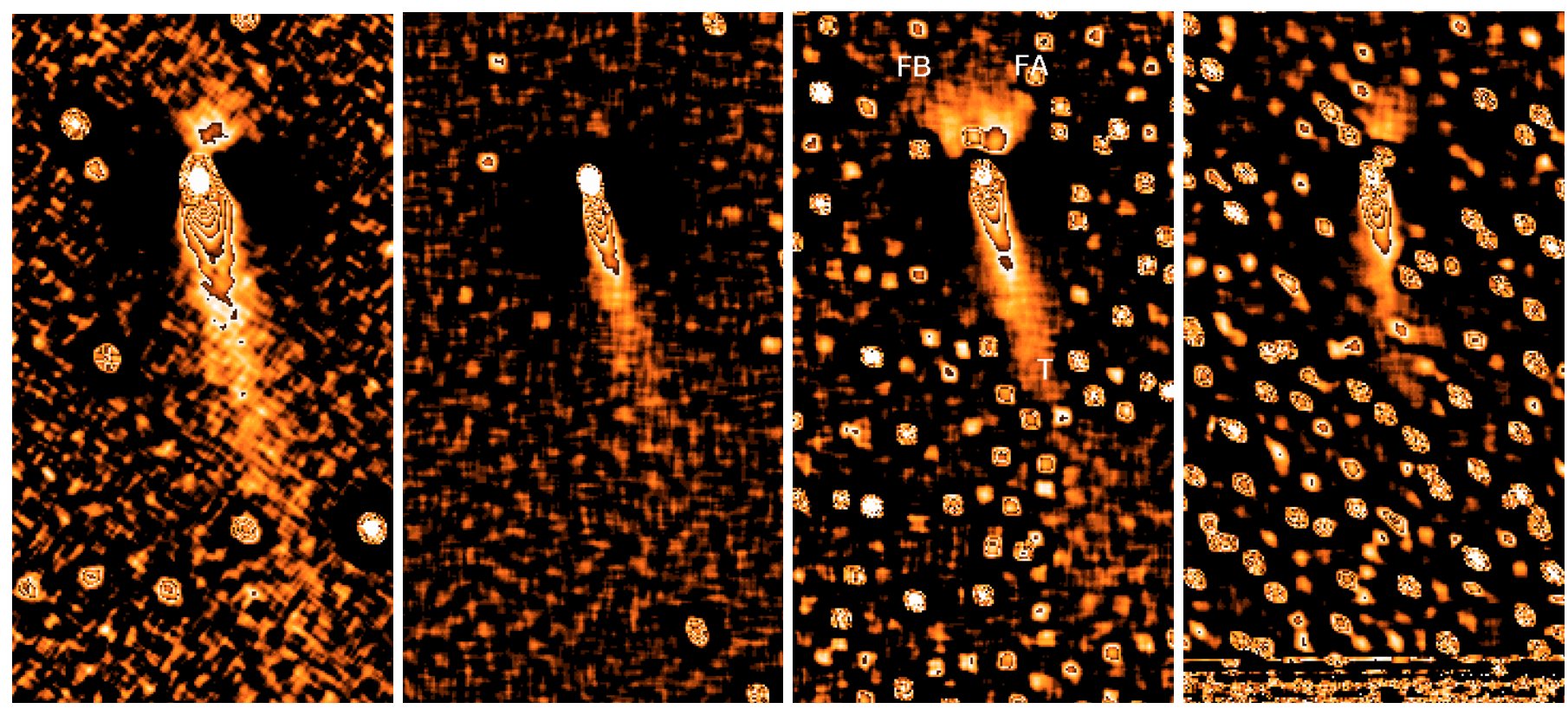

Fig. 8. Coma and tail structures in SW3 component C. The exposure series shows Laplace-filtered images, taken on 3, 4, 8, and 9 May 2006 (left to right). Orientation as on sky with Sun up. Field of view is $160 \times 295 \operatorname{arcsec}$ equivalent to $14000 \times 25700 \mathrm{~km}$ on $3 \mathrm{May}, 13200 \times 24200 \mathrm{~km}$ on 4 May, $11000 \times 20200 \mathrm{~km}$ on 8 May, and $10600 \times 19400 \mathrm{~km}$ on 9 May 2006 at the distance of component C. In the figure panels the various coma structures are identified as listed in Table 3.

antisolar direction. As expected, it appears to be curved slightly counterclock-wise.

Coma structures in component B (Table 4 and Fig. 9): Component $\mathrm{B}$ displayed a greater variability in the coma isophotes, i.e. more elongated elliptical on 3 and 4 May and irregular and wider on 8 and 9 May 2006 (see Sect. 3.1). The enhanced images (Fig. 9) show - besides the anti-sunward pointing dust tail (labeled T1) - a short (3000-4000 km) straight and sunward-oriented coma fan on 8 and 9 May (labeled FA and FB), a very short (250-500 km) spiky sunward structure on 3 and 4 May (labeled SP), a complex and variable pattern of arcs, 

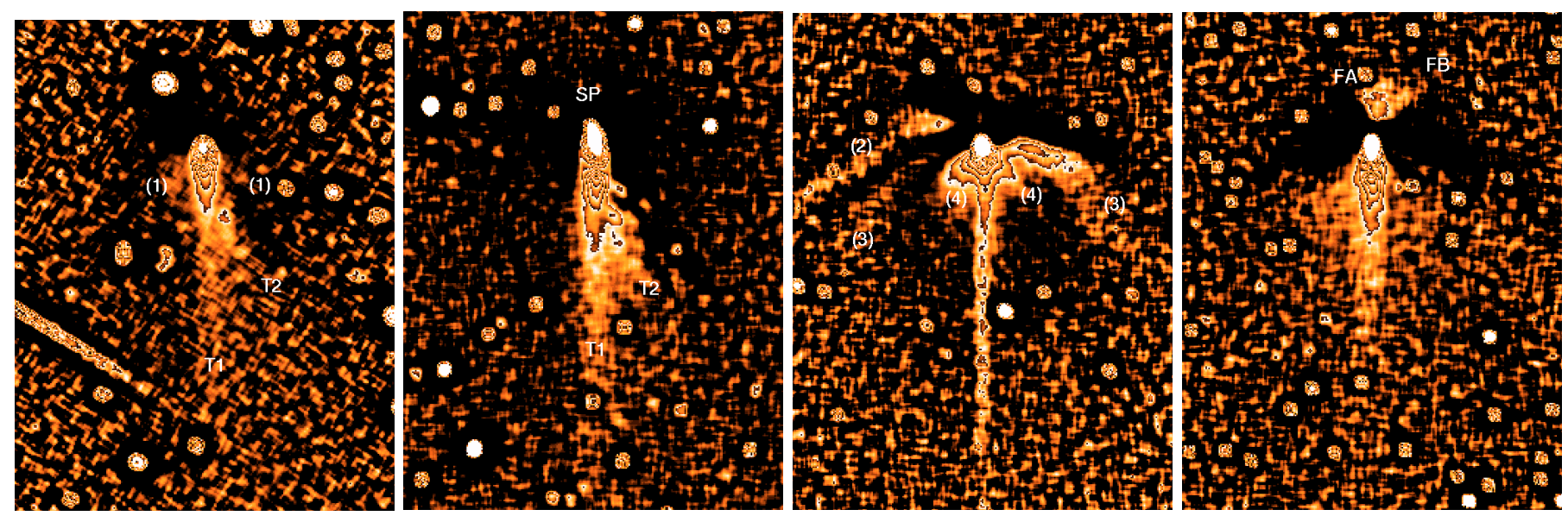

Fig. 9. Coma and tail structures in SW3 component B. The exposure series shows Laplace-filtered images, taken on 3, 4, 8, and 9 May 2006 (left to right). Orientation as on sky with Sun up. Field of view is $230 \times 300 \operatorname{arcsec}$ equivalent to $20600 \times 27000 \mathrm{~km}$ on $3 \mathrm{May}, 19700 \times 25700 \mathrm{~km}$ on 4 May, $15300 \times 20000 \mathrm{~km}$ on 8 May and $14400 \times 18800 \mathrm{~km}$ on 9 May 2006 at the distance of component B. In the figure panels, the various coma structures are identified as listed in Tables 3 and 4.

extending sideways from the dust tail on 3, 8 and 9 May 2006 (labeled 1, 2, 3, 4) and a secondary dust tail on 3 and 4 May 2006 (labeled T2). Our image series only allows us to conclude that the variability of the structures in component $\mathrm{B}$ happens on time scales of days.

The sunward fan in B (FA-FB) could have a similar origin as explained for the analog structure seen in component $\mathrm{C}$. However, since it is only seen during our last two observing nights, it is not possible to firmly conclude or to constrain any of the related nucleus parameters. Our pictures need to be interpreted in the context of a larger image series that displays the time evolution of the fan structure over a longer orbit arc.

The coma arcs - one pair each $(1,3)$ on 3 and 9 May 2006 plus two pairs ( 3 and 4) plus a single-sided one (2) on 8 May 2006 - have the typical appearance associated with fragmentation events (see Boehnhardt 2004). Similar arcs occur within a short time (1-2 days) after the fragmentation of cometary nuclei, and they fade away and become diffuse within a few days after their first appearance. They are believed to mark the collision zone of the comae of the fragments, hence the vertex of the arc is located between the two respective sub-nuclei. In the case of the SW3 arcs it is between about 400 and $2000 \mathrm{~km}$ projected distance from the nucleus of component B. If this interpretation is correct for the SW3 component B images, we can conclude that the arcs seen 3 May and 8+9 May are produced by different fragmentation events of component B. From lightcurve measurements of amateurs, rapid short-duration brightness increases are reported on 2 and 8 May 2006, which could be related to break-up events of nucleus B. The arc pattern on 8 and 9 May 2006 suggests that two new fragments might be produced on the tailward side of the nucleus. The single-sided arc on the sunward side might be related to yet another sub-nuclei of component B. In particular, the spiky short structure seen on 3 and 4 May 2006, could have hosted a sub-nucleus that was produced during a possible break-up event on 2 May 2006 or earlier and that was slowly drifting into the sunward coma hemisphere. The small and only slowly growing extension measured for the spiky structure (SP) supports this scenario. On 8 May 2006 this subnucleus might have fragmented, now leaving the single-sided arc to mark the event in the sunward coma hemisphere. No spiky structure is seen in our images of 8 and 9 May 2006, although its detection should have been possible assuming a similar brightness to that of 3 and 4 May 2006.
On 9 May 2006 the main arcs on the tailward side started to diffuse and spread out over a wider area in the dust tail region. The presence of the arcs as signatures of nucleus fragmentation in component $\mathrm{B}$ indicates that besides dust from the normal activity of $\mathrm{B}$, a considerable amount of additional gas is also released by the new fragments and that boulders and more dust are likely to be produced by the break-up events, namely in the tailward coma hemisphere.

Finally, we note that, on 3 and 4 May 2006 (maybe marginally also on 8 May 2006), a diffuse band of dust (T2) was seen westward of the main axis of the dust tail (T1) of component B. This band seemed to fade away quickly with time, since it was only marginally detected on 8 May 2006. The band follows approximately a zero velocity synchrone with emission time around 25 April 2006 ( \pm 3 days). It is in this tail region where 2-3 fragments are detected in our images of 3 and 4 May 2006, departing tailward from the main nucleus of component B. The diffuse appearance of the T2 tail suggests considerable dust release by these (and likely more unseen) fragments. The fragments themselves might be related to the outburst, making it a break-up event, as reported by amateurs on 24 April 2006.

\subsection{Polarimetric imaging}

Phase-angle dependence: The polarization of cometary dust shows strong phase-angle dependence (Jockers 1997a, and references therein). The phase angle of our targets changed during the observing period (Table 1). Therefore, our first objective is to see how the mean degree of linear polarization of both fragments, measured in their inner comae, changes with phase angle. For this purpose we applied Eq. (3) to the fluxes measured in the ordinary and extraordinary beams, here averaged in rectangular apertures of size $700 \times 4000 \mathrm{~km}$ and centered on the central brightness peaks of the components. The results are shown in Fig. 10. They are compared with the "standard phase curve of polarization" for dusty comets in the red continuum. The latter dependence was found empirically from polarimetric measurements of a number of "dusty" comets and is given by (Lumme \& Muinonen 1993; Kiselev 2003):

$P(\alpha)=32.049 \times \sin (\alpha)^{0.825} \times \cos (\alpha / 2)^{0.425} \times \sin \left(\alpha-21.8^{\circ}\right) .(4)$ 


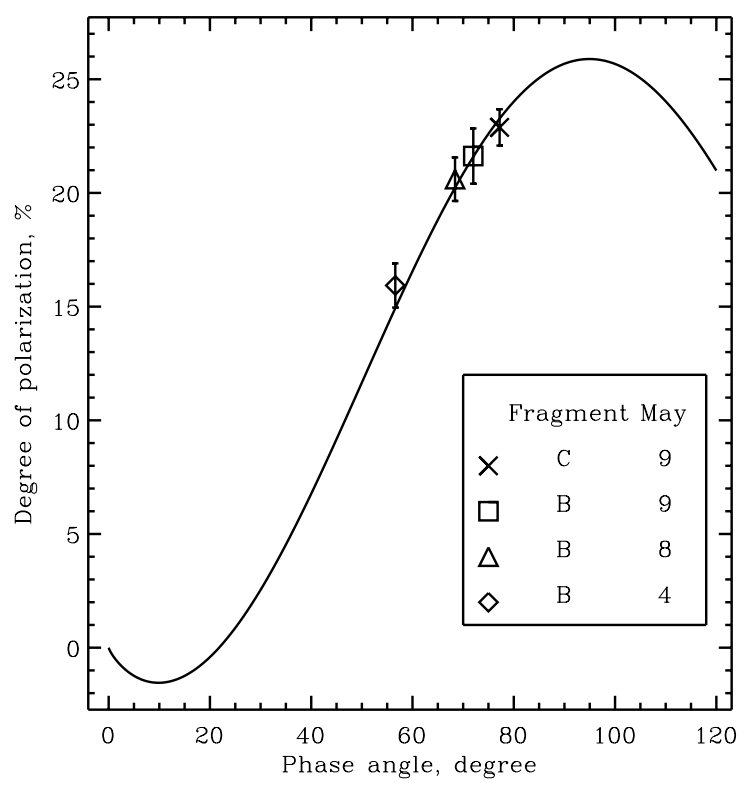

Fig. 10. Phase-angle dependence of the linear polarization measured in a rectangular region of size $700 \times 4000 \mathrm{~km}$, centered on SW3 components B and C. The continuous line represents the "standard phase dependence for dusty comets", given by Eq. (4).

Our measurement of the polarization degree of SW3 components $\mathrm{B}$ and $\mathrm{C}$ shows a good agreement - within the errors with the empirical relationship.

Polarization in Sun-tail direction: To analyze the dependence of polarization on cometocentric distance, we derived mean profiles of linear polarization along the solar-antisolar line. Subimages of full width of $700 \mathrm{~km}$, centered on the components, were extracted from the polarimetric images and averaged in a direction perpendicular to the sun-comet line. The individual profiles were aligned and averaged. The resulting mean polarization profiles are shown in Fig. 11. The vertical bars indicate the $1 \sigma$ error as calculated from the scatter between the individual profiles at a given cometocentric distance. The dotted horizontal lines in this figure represent the "standard" level of polarization given by Eq. (4) for the corresponding phase angle.

In most cases, the polarization profiles are characterized by a maximum close to the position of the brightness peak in the coma of the respective component and by a decrease in both, anti-solar and solar, directions. The observed maxima are close to the "standard" levels (components B and C on May 9) or exceed them by about $1 \%$ (component B on May 4 and 8). We note that on 8 May 2006 the position of the maximum polarization in B was shifted from the component's coma peak by about $1000 \mathrm{~km}$ into sunward direction. At the same time, the usual trend to a gradual decrease in the polarization degree with increasing cometocentric distance has changed to a disturbed "wavy" behavior. We also note that component $\mathrm{C}$ shows more symmetric slopes of the polarization curve with cometocentric distance with gradients in the Sun direction similar to B on 4 and 9 May 2006, but a steeper one than B in the tail direction.

\section{Discussion of the polarization results}

After the pioneering work by Eaton et al. (1988) on comet 1P/Halley, several other comets have been observed by means of polarimetric imaging. A list of these observations and their

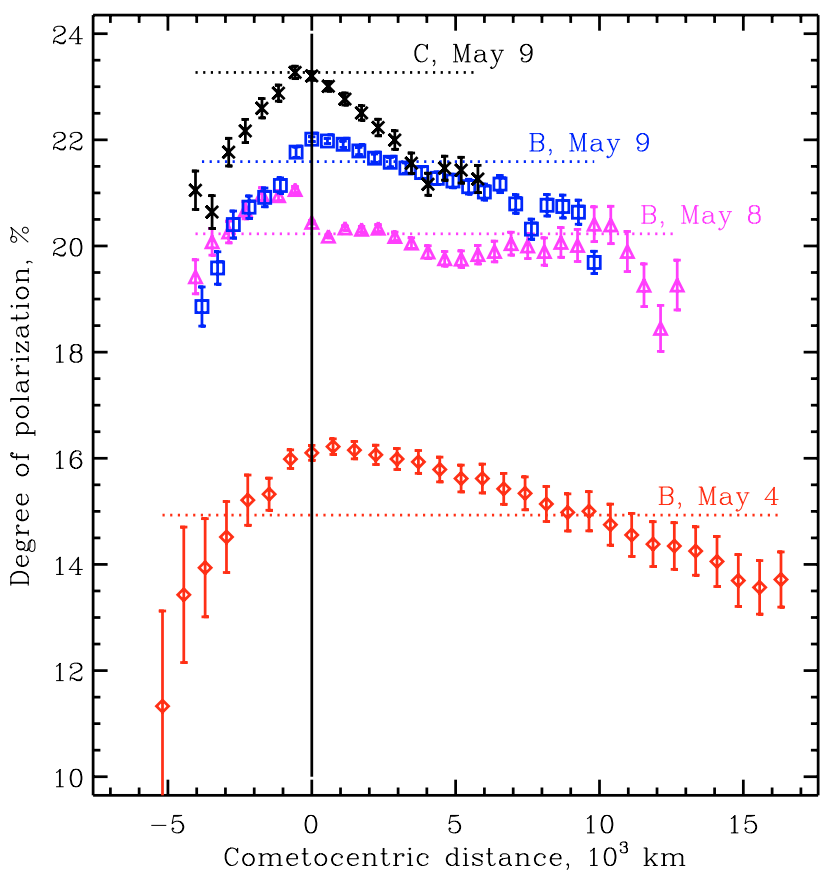

Fig. 11. Degree of linear polarization measured in the coma of SW3 component $\mathrm{B}$ on 4,8 , and 9 May 2006, and of component $\mathrm{C}$ on 9 May 2006. The profiles are taken along the sun-comet line. Sun direction is to the left. The broken lines indicate the polarization level according to Eq. (4).

analysis can be found in the review paper by Kolokolova et al. (2004). Recently, Jewitt (2004) and Jockers et al. (2005) have presented results of the spatial distribution of polarization in the inner coma of comet $2 \mathrm{P} /$ Encke. They have shown that, according to its polarization, comet $2 \mathrm{P} /$ Encke should belong to the hypothetical class of "dusty" comets as defined by Levasseur-Regourd et al. (1996). Jewitt (2004) derived an increase in the mean polarization with distance from the nucleus up to $\gtrsim 1000 \mathrm{~km}$. He concludes that this enhancement must reflect some systematic change in the nature of the dominant scatterers when moving outwards in the coma, and consideres the disaggregation of porous, composite grains as a likely mechanism. In contrast, Kiselev et al. (2004) found a high polarization in the near-nucleus region of $2 \mathrm{P} /$ Encke and a strong decrease with increasing distance. This behavior was explained by the depolarizing influence of gas contamination in the broadband filters used in these observations.

Often a polarization excess seen in the images appears with the presense of discrete structures (jets, fans, arcs) in the ambient coma (Tozzi et al. 1997; Furusho et al. 1999; Hadamcik et al. 1997; Jockers et al. 1997). The correlation between activity and degree of polarization seems to be valid for both spatial and temporal variations. In the analysis presented here, we obtained an increased level of polarization in the inner comae of SW3 components B and C. This level refers to the "standard" value for dusty comets and is most probably due to the strongly peaked distribution close to the sources of dust emission in the comae. The measured excess polarization of component B on 4 May 2006 could be caused by recent and/or ongoing exceptional activity. Evidence for an increased activity comes from the arc structures revealed in images of component B on May 3 (see Sect. 3.2). Amateur astronomers reported brightening of the comet in early May, just before the beginning of our observing campaign. Actually, component B, which was rather stable 
during the 2001 return of the comet, started to be active months before its perihelion passage. High-resolution images obtained on 18 April with the Hubble Space Telescope ${ }^{1}$ show a swarm of minor fragments following the main component. Several days later, observations made with the ESO-VLT ${ }^{2}$ revealed a set of mini-comets in the neighbourhod of component $\mathrm{B}$, and also our own imaging observations provide some weak evidence of additional "knots" in the tail region of B. Obviously, fragment B was continously in an excited state during the 2006 apparition releasing smaller fragments from its surface, thus opening up areas of fresh material that served as local regions of increased activity. Possibly, some of these regions were able to produce only small structures that could not be revealed in our images, even after applying sophisticated enhancement techniques. But the existence of such hypothetical structures could explain the high degree of polarization measured in the inner coma of component B on 4 May 2006.

Except for the data for B on 8 May (our first observations after the outburst of this component), the other polarization profiles of $\mathrm{B}$, as well the profile of fragment $\mathrm{C}$, have one common characteristic; i.e. the polarization is decreasing gradually with increasing distance from the nucleus. One possible explanation for this behavior is the contamination of the dust polarization by gas emission bands. We have used a narrow-band filter designed for observations of the cometary continuum, but, as pointed out by Jockers et al. (2005), it is virtually impossible to find spectral continuum windows entirely free of cometary molecular emissions. In their high-resolution catalog (Brown et al. 1996) have measured 559 unidentified cometary emission lines, the brightest of them in the region 6000-7000 , where our filter has high transmission. The same authors strongly suspect that most lines are from the $\mathrm{NH}_{2}$ molecule (still not identified in high resolution laboratory spectra). The spatial distribution of neutral gases is shallower in comparison to the dust distribution, which increases the relative contribution of gas emissions to the total brightness of a comet (fragment) at greater cometocentric distances.

The role of possible gas contamination in the polarization measurements of the cometary dust coma can be assessed by means of numerical models. We investigated several combinations of the spatial distributions of dust and gas. The dust was assumed to follow a $\rho^{-\gamma}$ law, taking different values for the slope $\gamma$. In one of the cases, the distribution of neutral gas was calculated according to the Haser model (Haser 1957), using the parent and daughter scale lengths for $\mathrm{NH}_{2}$, the most probable contaminant at the wavelength of observation. In this case the polarization is reduced to the observed level, but the spatial distribution of the polarization differs from the observed one. The best reproduction of the polarization decrease and its spatial distribution was achieved assuming a uniformly distributed depolarizing medium around the fragments. Such a uniform distribution could be explained by an extended source of neutral gas in the coma. The swarm of subfragments (minicomets) produced by the continuous disintegration of the main fragments could possibly be the origin of the extended source. However, this requires the gas-todust ratio of the minicomets to be higher than that of the main source, fragment B. Although such a possibility cannot be ruled out, an alternative explanation could be variation in the dust particle properties with distance from the nucleus, i.e. with time of flight. Fragment B has been observed at ESO from the end of

\footnotetext{
1 http://hubblesite.org/newscenter/archive/releases/ 2006/18/fastfacts/

2 http://www . eso.org/public/outreach/press-rel/ pr-2006/phot-15-06.html
}

April to mid June 2006 (Tozzi et al. 2007). In these observations the linaer polarization has a maximum in a small area around the subfragments and decreases with increasing distance. The decrease is stronger in the direction of the Sun than in lateral directions. A decrease in polarization is seen also in the antisolar direction but with a shallower gradient. Thus, these observations confirm our result above.

The relative increase in polarization in the coma of fragment B during the outburst is an indication that particles released during quiet and active phases may have different properties. One explanation for the observed variability could be the increased relative amount of small particles produced during enhanced outburst-like (possible break-up) events of a comet. The excess of small particles can be produced by the appearance of many more and much smaller grains (preserving the shape of the particle size distribution). Alternatively, for a power-law particle size distribution, a decrease in the power index would result in an excess of small particles. Jewitt (2004) considers possible decrease in the mean size of the particles with distance as a likely explanation for the observed increase in polarization in the inner coma of comet 2P/Encke. Steady shrinkage of individual particles or fragmentation of aggregates could be possible mechanisms for reducing the mean size. The small particles could be released from the fragments themselves or from the surface of the numerous boulders possibly generated during the process of cascading fragmentation of component B and causing its brightness outbursts.

The size of the particles is only one of several parameters that can influence the polarization of a dust coma. However, the size parameter alone may not represent the only answer to questions raised by observational facts; e.g., contradicting the scenario discussed in the previous paragraph, there is observational evidence for high polarization induced by large grains (Jockers et al. 2005). The interpretation of polarization and its variations in time and space should include a wide variety of dust characteristics, e.g. mineralogy, shape, and porosity, to mention some of them. The influence of these properties on the degree of linear polarization is reviewed by Kolokolova et al. (2004). Constraints for these parameters can be obtained by observations of cometary dust, performed simultaneously in different spectral ranges.

\section{Conclusions}

The broadband and polarimetric narrowband images of components $\mathrm{B}$ and $\mathrm{C}$ of the split comet $73 \mathrm{P} / \mathrm{Sch}$ wassmannWachmann 3 have shown that:

1. Component $\mathrm{C}$, considered the main fragment from the first splitting of the comet in 1995, was less active as compared to component $\mathrm{B}$, at least during the time of our observations (4 to 10 May 2006).

2. The coma of components B and C contained structures extending between several hundred to several thousand $\mathrm{km}$.

3. The polarization degree of the comae of components $\mathrm{B}$ and $\mathrm{C}$ inside distances $<1000 \mathrm{~km}$ is consistent with the "standard" phase curve for "dusty" comets.

4. The polarization of components $\mathrm{B}$ and $\mathrm{C}$ decreases with increasing distance in the coma, in both the sunward and tailward directions.

5. On 8 May 2006, during an observed outburst of fragment B, the decrease in polarization exhibited fluctuations with a tendency to compensate for the aforementioned decreasing 
trend. The fluctuations disappeared when fragment B was back to normal activity on 9 May 2006.

6. The outburst-like activity of fragment B was accompanied by significant changes in the coma-structure morphology. The arcs observed are considered indicators of further splitting events around the time of the outbursts.

7. An earlier outburst around 25 April 2006 has produced a secondary tail in component B that harbor mini-comets and show enhanced dust release.

Acknowledgements. T.B. greatly appreciates the opportunity to work as a visiting scientist at the Max-Planck-Institut für Sonnensystemforschung. Support by the staff of the National Astronomical Observatory-Rozhen during the observations is gratefully acknowledged. This research has made use of JPL's HORIZONS system and of NASA's Astrophysics Data System Bibliographic Services.

\section{References}

A'Hearn, M. F., Schleicher, D. G., Millis, R. L., Feldman, P. D., \& Thompson, D. T. 1984 , AJ, 89, 579

A'Hearn, M. F., Hoban, S., Birch, P. V., et al. 1986, Nature, 324, 649

Appenzeller, I. 1966, Z. Astrophys., 64, 269

Birtwhistle, P., \& Christensen, E. J. 2006, IAU Circ., 8692, 2

Boehnhardt, H. 2004, in Comets II, ed. M. Festou, H. U. Keller, \& H. Weaver (Univ. Arizona Press), 301

Boehnhardt, H., \& Birkle, K. 1994, A\&AS, 107, 101

Boehnhardt, H., Kaufl, H. U., Keen, R., et al. 1995, IAU Circ., 6274, 1

Boehnhardt, H., Holdstock, S., Hainaut, O., et al. 2002, Earth Moon and Planets, 90, 131

Bonev, T., Jockers, K., Petrova, E., et al. 2002, Icarus, 160, 419

Brown, M. E., Bouchez, A. H., Spinrad, A. H., \& Johns-Krull, C. M. 1996, AJ, 112, 1197

Christensen, E. J. 2006, IAU Circ., 8693, 2

Christensen, E. J., Hill, R. E., \& Kowalski, R. 2006, IAU Circ., 8685, 1

Colom, P., Crovisier, J., Biver, N., et al. 2006, in Semaine de l'Astrophysique Francaise, ed. D. Barret, F. Casoli, G. Lagache, A. Lecavelier, \& L. Pagani, SF2A-2006, 389
Combi, M. R. 1994, AJ, 108, 304

Crovisier, J., Biver, N., Bockelee-Morvan, D., et al. 1995, IAU Circ., 6227, 1

Crovisier, J., Bockelee-Morvan, D., Gerard, E., et al. 1996, A\&A, 310, L17

DiSanti, M. A., Anderson, W. M., Villanueva, G. L., et al. 2007, ApJ, 661, L101

Eaton, N., Scarrott, S. M., \& Warren-Smith, R. F. 1988, Icarus, 76, 270

Furusho, R., Suzuki, B., Yamamoto, N., et al. 1999, PASJ, 51, 367

Hadamcik, E., Levassuer-Regourd, A. C., \& Renard, J. B. 1997, Earth Moon and Planets, 78, 365

Haser, L. 1957, Bull. de la Société Royale des Sciences de Liege, 43, 740

Hasubick, W., \& Nagai, Y. 2006, IAU Circ., 8693, 3

Jewitt, D. 2004, AJ, 128, 3061

Jockers, K. 1997a, Earth Moon and Planets, 79, 221

Jockers, K. 1997b, Exp. Astron., 7, 305

Jockers, K., Rosenbush, V. K., Bonev, T., \& Credner, T. 1997, Earth Moon and Planets, 78, 373

Jockers, K., Kiselev, N., Bonev, T., et al. 2005, A\&A, 441, 773

Kiselev, N. N. 2003, Light scattering by dust particles of comets, asteroids, and circumstellar envelopes: Observations and Interpretation, Doctoral thesis, in russian, Kharkov, Ukraine

Kiselev, N. N., Jockers, K., \& Bonev, T. 2004, Icarus, 168, 385

Kolokolova, L., Hanner, M. S., Levasseur-Regourd, A.-C., \& Gustafson, B. Å. S. 2004, in Comets II, ed. M. Festou, H. U. Keller, \& H. Weaver (Univ. Arizona Press), 577

Lara, L., Rodrigues, P., Rodrigo, R., et al. 2006, IAU Circ., 8708, 1

Levasseur-Regourd, A. C., Hadamcik, E., \& Renard, J. B. 1996, A\&A, 313, 327

Lumme, K., \& Muinonen, K. O. 1993, Asteroids, Comets, Meteors, IAU Symp., 160, LPI Contributions, 810, 194

Sekanina, Z. 2005, International Comet Quarterly, 27, 225

Sekanina, Z., Jehin, E., Boehnhardt, H., et al. 2002, ApJ, 572, 679

Serkowski, K. 1974, in Planets, Stars, and Nebulae: Studied with Photopolarimetry, ed. T. Gehrels, IAU Colloq., 23, 135

Toth, I., Lamy, P., Weaver, H., et al. 2006, BAAS, 38, 489

Tozzi, G.-P., Cimatti, A., di Serego Alighieri, S., \& Cellino, A. 1997, Planet. Space Sci., 45, 535

Tozzi, G.-P., Bagnulo, S., Boehnhardt, H., et al. 2007, in AAS/Division for Planetary Sciences Meeting Abstracts, 39, 54.04

Villanueva, G. L., Bonev, B. P., Mumma, M. J., et al. 2006, ApJ, 650, L87

Weaver, H. A., Lisse, C. M., Mutchler, M. J., et al. 2006, in Bulletin of the American Astronomical Society, 38, 490 